\title{
Prescribing pattern of proton pump inhibitors among patients admitted to medical ward at King Abdulaziz University Hospital, Jeddah, Saudi Arabia
}

\author{
A retrospective study
}

Bodoor S. Al-Dosari, Pharm D, MSc, Bayan M. Binafeef, Pharm D, Samar A. Alsolami, Pharm D.

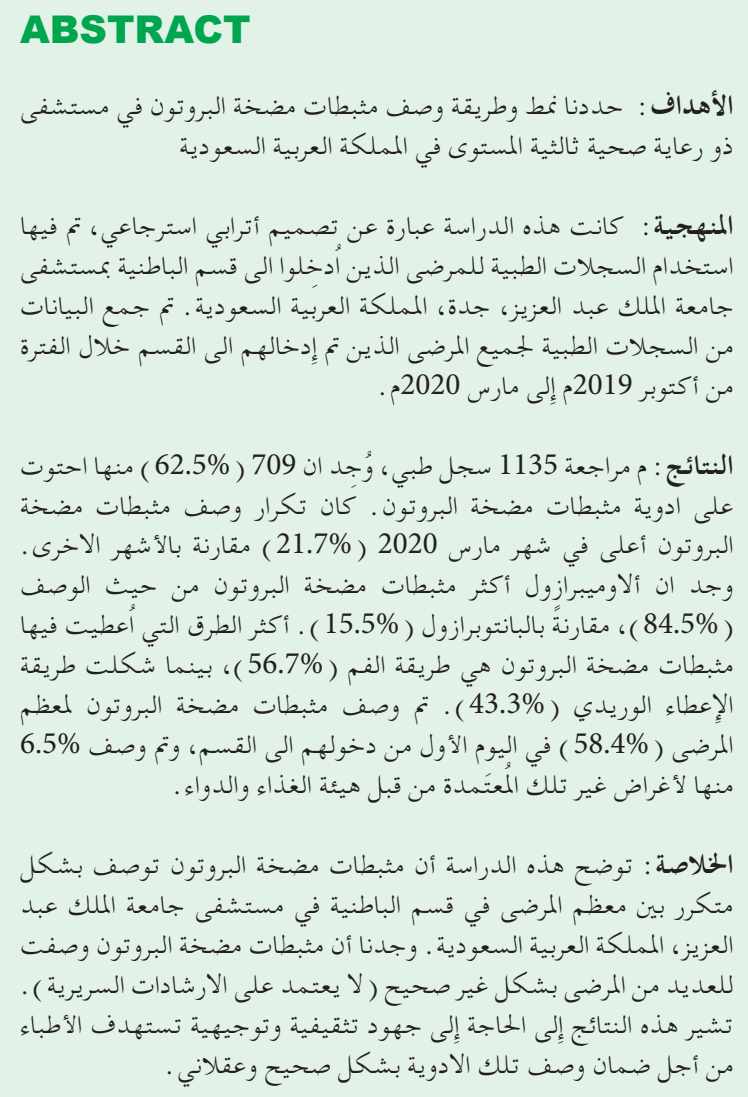

Objectives: To determine the prescription pattern of proton pump inhibitors (PPIs) at a tertiary hospital in Saudi Arabia.

Methods: This study was a retrospective cohort design using medical records at the medical ward of King Abdulaziz University Hospital (KAUH), Jeddah, Saudi Arabia. Data were collected from medical records of all patients admitted to the ward from October 2019 to March 2020.
Results: A total of 1135 medical records were retrieved, of which 709 (62.5\%) were prescribed PPIs. The frequency of PPIs prescribed was highest in March 2020 (21.7\%) relative to the remaining months. Omeprazole was the most prescribed PPI (84.5\%), compared to pantoprazole (15.5\%). The PPIs were commonly administered through oral $(56.7 \%)$, compared to intravenous route (43.3\%). The PPIs were prescribed to most patients (58.4\%) on the first day of admission, and 6.5\% were prescribed for indications other than those approved by the Food and Drug Administration.

Conclusion: This study demonstrates that PPIs are frequently prescribed among most patients at the medical ward of KAUH, Kingdom of Saudi Arabia. We found that the PPIs were prescribed to many patients inappropriately (not based on clinical guidelines). These results suggest the need for educational interventions among physicians on rational prescription.

Keywords: Rational drug use, proton pump inhibitors, ulcer, gastric acid, Saudi Arabia

Saudi Med J 2021; Vol. 42 (12): 1313-1319 doi: 10.15537/smj.2021.42.12.20210488

From the Department of Pharmacy, King Abdulaziz University Hospital, Jeddah, Kingdom of Saudi Arabia.

Received 22nd August 2021. Accepted 18th October 2021.

Address correspondence and reprint request to: Dr. Bodoor S. Al-Dosari, Pharmacy Department, King Abdulaziz University Hospital, Jeddah, Kingdom of Saudi Arabia.E-mail: bsaldosari@kau.edu.sa ORCID ID: https://orcid.org/0000-0002-2884-9423 
P roton pump inhibitors (PPIs) are drugs used in the management of dyspepsia, gastro-oesophageal reflux disease (GERD), peptic ulcer disease, and upper gastrointestinal bleeding. ${ }^{1}$ Proton pump inhibitors belong to a class of drugs also utilized as prophylactic agents in patients taking non-steroidal antiinflammatory drugs (NSAIDs). ${ }^{1}$ A common example of the PPIs approved by the United States Food and Drug Administration include omeprazole, esomeprazole, lansoprazole, rabeprazole, and pantoprazole. Although these drugs have been demonstrated to be the cornerstone in the management of gastric acid-related disorders, they differ in their pharmacokinetics, pharmacodynamics, and interactions with other medications. ${ }^{1}$ The most common side effects of PPIs are constipation, headache, abdominal pain, flatulence, and diarrhoea, which are mild and self-limiting. Moreover, chronic PPI therapy carries an increased risk of hip fractures, communityacquired pneumonia, Clostridium difficile infection, gastric carcinoids, and hypomagnesemia. ${ }^{2,3}$

Proton pump inhibitors are among the most frequently prescribed medications for ulcer management in clinical practice. ${ }^{4,5}$ A previous study reported that more than 113 million PPIs were prescribed worldwide in $2009 .{ }^{4}$ This estimate is expected to increase in the recent years. Over the past few years, PPI prescriptions have consistently increased in hospitals and ambulatory care settings. ${ }^{1}$ Given their common use in clinical practice, easy availability, high efficacy, and competitive marketing, PPIs are prone to be prescribed and misused. ${ }^{6}$ Many drug utilization studies have reported the widespread inappropriate use of PPIs, which could lead to serious and poor health outcomes. ${ }^{7-10}$ Therefore, research is needed to investigate the use of PPIs in clinical settings.

The prescription pattern of PPIs has been investigated worldwide, including the Middle East. ${ }^{7-12}$ Despite the introduction of a standard guideline for prescribing PPIs, findings from the previous studies indicated patients' need for appropriate prescription and use of PPIs. In Saudi Arabia, data related to PPI prescription are limited to those reported in the literature. The few available studies showed that PPIs were frequently prescribed to patients in tertiary hospitals, to inpatients and outpatients, and by community pharmacies. ${ }^{12-14}$

Disclosure. Authors have no conflict of interests, and the work was not supported or funded by any drug company.
However, more studies on the prescription pattern of PPIs are needed because of the increasing use of PPIs and need to improve drug safety in Saudi Arabia. ${ }^{15}$ Therefore, we aimed to assess the prescription patterns of PPIs in patients admitted to the medical wards (internal medicine) of King Abdulaziz University Hospital (KAUH), Jeddah, Saudi Arabia. Our secondary aim was to evaluate the appropriateness of prescribing PPIs to these patients.

Methods. A retrospective cohort study was conducted using data from the electronic medical records of all adult patients admitted to the medical wards (male and female wards) in KAUH, between October 2019 and March 2020. King Abdulaziz University Hospital is the largest tertiary care hospital in the Western region of Saudi Arabia. ${ }^{16}$ As of February 2021, the hospital has a capacity of 1,067 beds and 12 medical departments. ${ }^{16}$

Data from medical records that indicated PPI prescriptions were extracted and included in the study. Patient records that did not indicate any PPI prescription were excluded.

The authors designed a data collection tool based on the study aims, medical records, and discussion with an expert on the subject matter. The collection tool was tested for face validity among 30 personnel recruited from the department of medical records. It was presented to the participants, who were asked to provide review and feedback regarding the wordings, relevance, and appropriateness of the included items for achieving the study objectives. The tool was updated on the basis of the feedback received from the participants. The collected information included the patients' demographics (age and gender); clinical data; type, dose, route of administration, frequency of administration, commencement date of administration, and indication for administration of PPI; investigation and findings prior to diagnosis; use of PPIs before admission; duration of PPI use; and co-prescription with any of the following: clopidogrel, NSAIDs, steroids, calcium carbonate, or ferrous sulphate. Data collection and prescription validation were performed by 3 doctors (B.A., B.B., and S.A.) of whom one has a Master degree in clinical pharmacy and is a board certified critical care pharmacist (B.A.).

The PPI utilisation pattern was evaluated by dividing the number of patients who received PPIs by the total number of patients admitted to the ward throughout the study period. The prescription pattern of each PPI was determined by calculating the percentage of the PPI prescribed with respect to the total number 
of PPIs prescribed to the patients during the study period. We also assessed the appropriateness of the PPI prescribed in the patients by comparing the regimen of the prescribed PPI (right dose, frequency, route, time, diagnosed indication, and drug interaction) with the recommendations provided in the UpToDate and Micromedex online databases and National Institute for Health and Care Excellence guidelines for PPI use. ${ }^{17-19}$ The guidelines and recommendations provide dosage information on PPIs for the management of GERD (erosive and non-erosive), peptic ulcer disease, functional dyspepsia, and severe esophagitis. The research protocol was reviewed and approved by the research ethics committee of KAUH (reference No. 368-20). Owing to the retrospective nature of this study, consent from the participants was not required. However, we ensured the confidentiality of their data through the collection of de-identified information from their records.

Statistical analysis. Data analysis was performed using IBM SPSS Statistics Version 25.0 for Windows (IBM Corp., Armonk, NY). Data were analyzed using descriptive statistics. Continuous data were expressed as mean (standard deviation [SD]) or median (interquartile range $[\mathrm{IQR}]$ ); and categorical data, as percentages and frequencies. Charts were created using GraphPad Prism version 5.01 for Windows (GraphPad Software Inc., San Diego, CA, www.graphpad.com).

Results. A total of 1135 medical records were retrieved at KAUH during the study period. We identified the records of $709(62.5 \%)$ patients who received PPIs during admission to the medical ward. Of these patients, $362(51.1 \%)$ were male and 347 (48.9\%) were female, and 365 (51.5\%) were aged $\geq 60$ years. The patients' demographic characteristics are summarized in Table 1.

Prescription pattern of PPIs. An age-wise decline in the number of PPI prescriptions was observed, in which the highest prescription rates were recorded in patients aged $>60(51.5 \%)$ years and lowest prescription rates were recorded in patients aged $<20(2.3 \%)$ years. Figure 1 demonstrates the age-wise pattern of PPI prescription pattern during the study period. The frequency of PPI prescription in the patients was observed during the study period. It was highest in March 2020 (21.7\%) compared with the other months in the same year. Of all the patients, $62 \%$ received PPI prescriptions per month (over the 5 -month period). Figure 2 demonstrates the PPI prescription pattern during the study period.

We found only 2 types of PPIs (omeprazole and pantoprazole) prescribed to the patients during the
Table 1 - Demographic characteristics of the patients (N=709).

\begin{tabular}{lc}
\hline Variables & $\mathbf{n}(\%)$ \\
\hline Gender & \\
Female & $347(48.9)$ \\
Male & $362(51.1)$ \\
Age group (years) & \\
$<20$ & $16(2.3)$ \\
$20-39$ & $127(17.9)$ \\
$40-59$ & $201(28.3)$ \\
60 years and above & $365(51.5)$ \\
Indication for proton pump inhibitors & \\
Eradication of Helicobacter pylori & $3(0.4)$ \\
Gastroesophageal reflux disease & $5(0.7)$ \\
Peptic ulcer disease & $18(2.5)$ \\
Peptic ulcer prophylaxis & $6(0.8)$ \\
Stress ulcer prophylaxis & $7(1.0)$ \\
Heartburn & $3(0.4)$ \\
Not documented & $617(87.0)$ \\
Others & $46(6.5)$ \\
\hline
\end{tabular}

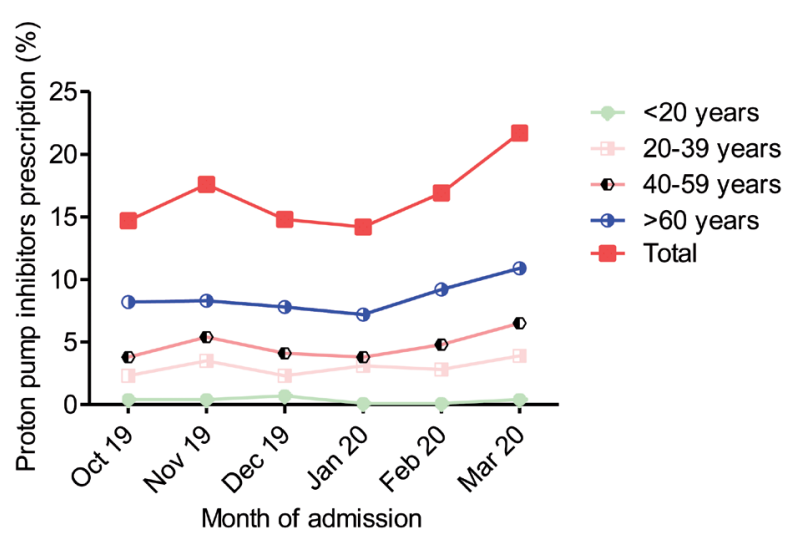

Figure 1 - Age-wise prescription pattern of proton pump inhibitors from October 2019 to March 2020 at the medical ward of King Abdulaziz University Hospital, Jeddah. Saudi Arabia.

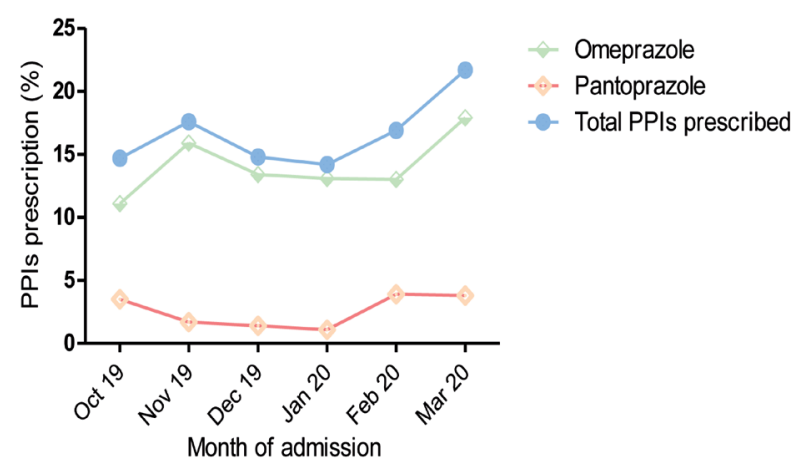

Figure 2 - Proton pump inhbitors utilization pattern from October 2019 to March 2020 at the medical ward of King Abdulaziz University Hospital. PPIs: proton pump inhibitors 
5-month study period. Omeprazole (84.5\%) was determined to be more frequently prescribed than pantoprazole (15.5\%). In terms of dosage, omeprazole was prescribed more frequently at $40 \mathrm{mg}(81.9 \%)$, followed by $20 \mathrm{mg}(15.1 \%)$, and then $80 \mathrm{mg}(2.7 \%)$. The frequency of oral route prescriptions was relatively higher $(56.7 \%)$ than that of the intravenous route (43.3\%) and mostly ordered once daily (76.3\%; Table 2). The PPIs were prescribed to most of the patients $(58.4 \%)$ on the first day of admission to the medical ward. Only $0.3 \%$ were prescribed following the diagnostic investigation to confirm the indication. The indication of use was documented in $13 \%$ of the patients. However, for $87 \%$ of the patients, indication for PPI use was not reported.

The most common duration of PPI administration during admission was $>1$ week in $51 \%$ of the admitted patients. Proton pump inhibitors were also prescribed in $48 \%$ of the patients at hospital discharge, of whom 92\% use PPIs for 1 to 3 months (Table 3). The medical records of all the patients indicated that $48.8 \%$ of the patients were using PPIs before their admission to the hospital (as outpatients). The medication histories showed that $16.2 \%$ of the patients used PPIs for 1 to 3 months and $13 \%$ used PPIs for $>1$ year.

Co-prescription with PPIs. The most frequently coprescribed medication with PPIs was aspirin (33.6\%). The other drugs co-prescribed with PPIs included prednisolone, meloxicam, clopidogrel, ferrous sulphate,

Table 2 - Prescription pattern of proton pump inhibtors at the medical ward of King Abdulaziz University Hospital, Jeddah, Kingdom of Saudi Arabia.

\begin{tabular}{|c|c|c|c|}
\hline Variables & Omeprazole & Pantoprazole & Total PPIs prescribed \\
\hline \multicolumn{4}{|c|}{ Route of administration } \\
\hline Intravenous & $260(36.7)$ & $46(6.6)$ & $307(43.3)$ \\
\hline Per oral & $339(47.8)$ & $63(8.9)$ & $402(56.7)$ \\
\hline \multicolumn{4}{|l|}{ Dose } \\
\hline $120 \mathrm{mg}$ & $1(0.1)$ & $0(0)$ & $1(0.1)$ \\
\hline $20 \mathrm{mg}$ & $94(13.2)$ & $13(1.8)$ & $107(15.1)$ \\
\hline $40 \mathrm{mg}$ & $485(68.4)$ & $96(13.5)$ & $581(81.9)$ \\
\hline $8 \mathrm{mg}$ & $1(0.1)$ & $0(0)$ & $1(0.1)$ \\
\hline $80 \mathrm{mg}$ & $18(2.5)$ & $1(0.1)$ & $19(2.7)$ \\
\hline \multicolumn{4}{|c|}{ Frequency of administration } \\
\hline b.i.d & $132(18.6)$ & $21(3.0)$ & $153(21.6)$ \\
\hline Inf & $11(1.6)$ & $0(0)$ & $11(1.6)$ \\
\hline o.d & $452(63.8)$ & 89 (12.6) & $541(76.3)$ \\
\hline t.i.d & $3(0.4)$ & $0(0)$ & $3(0.4)$ \\
\hline \multicolumn{4}{|c|}{$\begin{array}{l}\text { Values are presented as number and percentage (\%). PPIs: proton pump } \\
\text { inhibtors, b.i.d: twice daily, Inf: infusion. o.d: once daily, t.i.d: } 3 \text { times } \\
\text { daily. }\end{array}$} \\
\hline
\end{tabular}

steroids, and calcium carbonate. Patients who were given clopidogrel more frequently received pantoprazole (42\%) than omeprazole (Figure 3).

Inappropriate PPI prescription. In this study, 6.5\% of the PPIs prescribed were indicated for complaints other than those approved by the reference guidelines used for this study. Such complaints included coffee ground emesis, nausea and vomiting, epigastric pain, gastritis, irritable bowel syndrome, bleeding, oesophageal varices, stomach cancer, and melena with no gastrointestinal bleeding.

Discussion. Currently, there is lack of information in the literature describing the prescription pattern of PPIs in patients admitted to the medical wards of KAUH. We found that PPIs were prescribed in approximately $60 \%$ of the patients. The frequency of PPI prescriptions over the 5-month period was highest in March 2020 among the other months in the same year. Omeprazole and pantoprazole were the most frequently prescribed PPIs among the patients. Proton pump inhibitors were mainly prescribed on the first day of admission at the medical ward in approximately $58 \%$ of the patients. We also found that $6.5 \%$ of the PPI prescriptions were inappropriate. Therefore, our findings showed that PPIs are frequently prescribed to most patients at the medical wards of KAUH. The inappropriate prescriptions suggest the need for prescribers to adhere to official monographs and guidelines to ensure rationale prescription and safe drug use in Saudi Arabia.

We found that PPIs were prescribed almost equally in terms of frequency for both male and female patients, most of whom were aged 60 years. This result is consistent with that of a similar study conducted at a

Table 3 - Duration of proton pump inhibitors (PPIs) used among the participants $(\mathrm{N}=790)$.

\begin{tabular}{lc}
\hline Duration of PPI use & $\mathbf{n}(\%)$ \\
\hline $\begin{array}{l}\text { During admission } \\
\text { 1-3 days }\end{array}$ & $122(17)$ \\
4-7 days & $104(14)$ \\
>7 days & $365(51)$ \\
1 month & $49(6.9)$ \\
>1 month & $69(9.7)$ \\
At point of discharge & \\
6-29 days & $24(6.9)$ \\
1-3 months & $317(92)$ \\
4-6 months & $4(1.16)$ \\
12 months & $1(0.2)$ \\
\hline
\end{tabular}




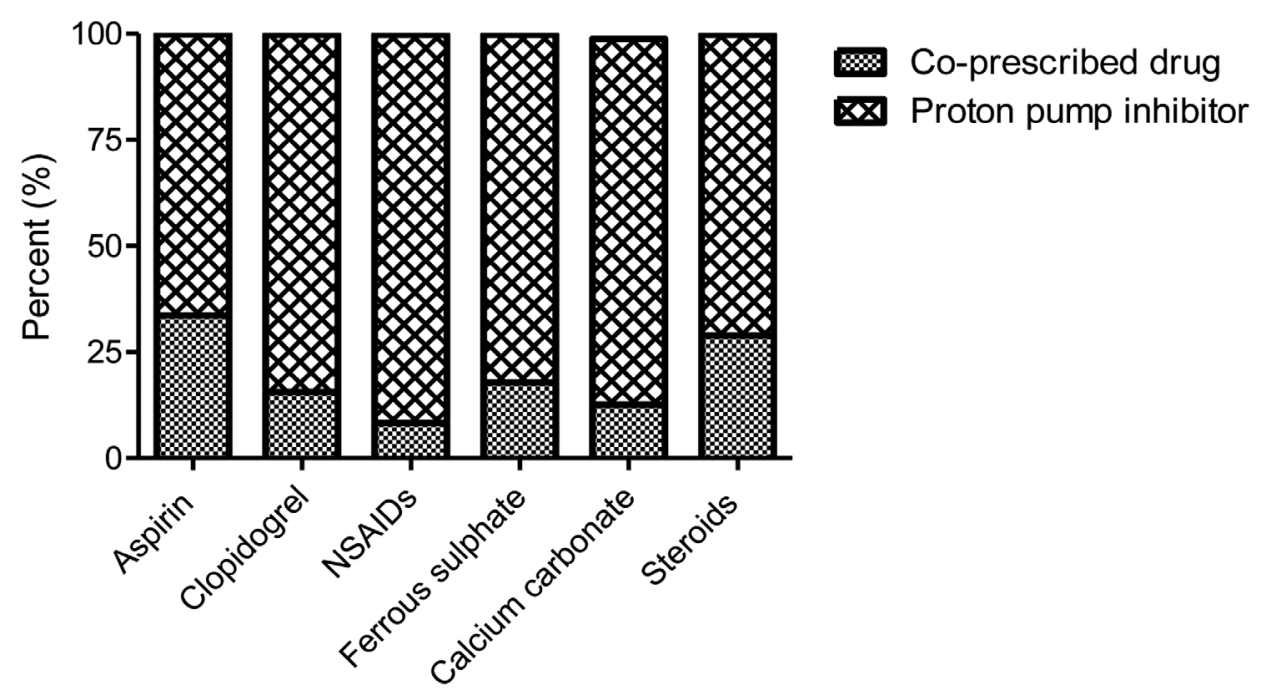

Figure 3 - Co-prescription pattern of proton pump inhibitors with other medications.

teaching hospital in South India, ${ }^{20}$ supporting the equal prevalence of ulcer between the genders. ${ }^{21}$ However, a previous study conducted at Aseer Central Hospital, Abha, Saudi Arabia, ${ }^{13}$ reported a higher proportion of female patients who received PPIs. The variation could be due to the differences in study aims and target population. The study excluded patients who were previously taking PPIs or those already diagnosed with gastrointestinal disorders. ${ }^{13}$

In this study, omeprazole and pantoprazole were the most frequently prescribed types of PPI in the medical ward, with the former having a higher prescription frequency than the latter. The common use of these drugs in the present study may reflect prescribers' good knowledge of omeprazole and the widespread use and availability of the 2 drugs in Saudi Arabia, as reported in similar studies in Saudi Arabia. ${ }^{13}$ This finding shows that both older (omeprazole) and newer PPIs (pantoprazole) are utilized in the facility.

Although the oral route of administration was the most recommended in this setting, we found that PPIs were given to approximately $40 \%$ of the patients through the intravenous route. The same experience was reported by a previous study at a tertiary hospital in Saudi Arabia. ${ }^{13}$ However, a retrospective cohort study at a teaching hospital in the United States reported that $71 \%$ of patients received pantoprazole intravenously. ${ }^{22}$ Although no consensus has been reached among most gastroenterology associations, ${ }^{23}$ the outcomes of some randomized controlled trials supported the clinical benefits of PPI administration through the intravenous route in acute cases of acid-related disorders. ${ }^{23-25}$ Intravenous use of PPIs in this setting may be described as inappropriate, but this theory requires further study to investigate the suitability of intravenous administration of pantoprazole, considering the specific circumstances of patients and indications for administration. Furthermore, interventions to improve rational drug prescription are thus needed among prescribers to improve patient safety.

Half of the patients in this study received PPI on their first day of hospital admission, but the indication for PPI use was rarely documented in their medical records. In addition, we found that diagnostic investigation was conducted in only 5.8\% of the patients before PPIs were prescribed, and many patients received PPIs for indications other than those recommended by the guidelines. These results suggest that PPIs are used inappropriately in a significant number of patients and a potential cause of error during medication reconciliation at the time of transition of care for patients in the hospital setting. Our findings are consistent with those of previous studies reported by Akram et $\mathrm{al}^{26}$ and Madi et al. ${ }^{8}$

The duration of PPI administration during admission was $>1$ week in half of the patients, and $92 \%$ of the patients received PPIs for 1 to 3 months as a take-home medication after hospital discharge. These findings are alarming, as prolonged use of PPIs could lead to adverse events. ${ }^{1}$ This inappropriate prescription pattern of PPIs with no clear indication is probably due to the many physicians who continue to prescribe PPIs, considering 
them as safe and harmless without assessment of the risk of the side effects of long-term therapy.

We found co-prescriptions of PPIs with potentially interacting drugs such as ferrous sulphate and clopidogrel. Proton pump inhibitors could decrease the absorption of minerals and vitamins, including ferrous sulphate, which leads to sub-optimal efficacy. ${ }^{27-29}$ Similarly, in a randomized clinical crossover trial that involved patients with myocardial infarction, omeprazole-clopidogrel co-prescription was reported to show significant pharmacodynamic interactions that resulted in reduced antiplatelet efficacy.

Practical implications of the study. This is the first study to describe the PPI prescription pattern at KAUH, Jeddah, Saudi Arabia. Our findings suggest the need for physicians to adhere to the current guidelines for prescribing PPIs to prevent the increase of the burden from drug-related complications. ${ }^{30}$

An educational program on the proper use and side effects of the long-term use of PPIs, development of a protocol for prescribing PPIs for medically admitted patients, and involvement of medication safety officers in monitoring the prescription patterns of PPIs should be implemented to control the use of PPIs in hospital settings. ${ }^{8}$

We observed much missing information in the retrieved medical records. Therefore, proper documentation of patient profiles and medication histories and details about the drugs are needed. ${ }^{31}$

Study limitations. The retrospective design of the present study are associated with inherent shortcomings such as missing data. Provided this limitation, many patients might have received PPIs for a recommended indication that was not reported.

In conclusion, our study demonstrate that PPIs were frequently prescribed to most patients at the medical wards of KAUH. We found that PPIs were prescribed inappropriately (not based on clinical guidelines) to many patients.

We suggest the implementation of educational interventions for physicians on rational prescription and adherence to the recommendations in official monographs and to the guidelines for PPI use.

Acknowledgment. We would like to thank Scribendi (https:// www.scribendi.com) for English language editing.

\section{References}

1. Savarino V, Marabotto E, Zentilin P, Furnari M, Bodini G, De Maria C, et al. Proton pump inhibitors: use and misuse in the clinical setting. Expert Rev Clin Pharmacol 2018; 11: 1123-1134.
2. Fossmark R, Martinsen TC, Waldum HL. Adverse effects of proton pump inhibitors-evidence and plausibility. Int J Mol Sci 2019; 20: 5203.

3. Liu Y, Zhu X, Li R, Zhang J, Zhang F. Proton pump inhibitor utilisation and potentially inappropriate prescribing analysis: insights from a single-centred retrospective study. BMJ Open 2020; 10: e040473.

4. Katz MH. Failing the acid test: Benefits of proton pump inhibitors may not justify the risks for many users. Arch Intern Med 2010; 170: 747-748.

5. Hálfdánarson ÓÖ, Pottegård A, Björnsson ES, Lund SH, Ogmundsdottir MH, Steingrímsson E, et al. Proton-pump inhibitors among adults: a nationwide drug-utilization study. Therap Adv Gastroenterol 2018; 11: 1756284818777943.

6. Savarino V, Marabotto E, Zentilin P, Furnari M, Bodini G, De Maria C, et al. The appropriate use of proton-pump inhibitors. Minerva Medica 2018; 109: 386-399.

7. O'Mahony L, Yelverton E. Prescribing of proton pump inhibitors in an Irish general practice. Ir Med J 2019; 112: 932.

8. Madi L, Ahmed Elhada AH, Alrawashdeh H, Ahmed A Prescribing pattern of proton pump inhibitors in Qatar rehabilitation institute: A retrospective study. J Res Pharm Pract 2019; 8: 101-104.

9. Jarbøl DE, Lykkegaard J, Hansen JM, Munck A, Haastrup PF. Prescribing of proton-pump inhibitors: Auditing the management and reasons for prescribing in Danish general practice. Fam Pract 2019; 36: 758-764.

10. Kazberuk M, Brzósko S, Hryszko T, Naumnik B. Overuse of proton pump inhibitors and its consequences. Postepy Hig Med Dosw (Online) 2016; 70: 1112-1116.

11. Gamelas V, Salvado V, Dias L. Prescription Pattern of Proton Pump Inhibitors at Hospital Admission and Discharge. GE Port J Gastroenterol 2019; 26: 114-120.

12. Ali MD, Ahmad A. A retrospective study on prescribing pattern and cost analysis of proton-pump inhibitors used among adults of Saudi Arabia. J Pharm Health Serv Res 2020; 11: 343-347.

13. Alzahrani MA, Al Ghamdi BA, Al ahmari HS, Alqahtani EA, Saleh AG, Ahmed MEK. The Prescribing Pattern of Proton Pump Inhibitors by Non-gastroenterologists in a Tertiary Teaching Hospital in the Kingdom of Saudi Arabia. JAASP 2016; 5: 341-345.

14. Alhossan A, Alrabiah Z, Alghadeer S, Bablghaith S, Wajid S, Al-Arifi M. Attitude and knowledge of Saudi community pharmacists towards use of proton pump inhibitors. Saudi Pharm J 2019; 27: 225-228.

15. Al-Worafi YM, ed. Drug safety in Saudi Arabia. In: Drug Safety in Developing Countries. Elsevier; 2020. pp. 407-417.

16. King Abdulaziz University Hospital. About Hospital. [Updated 2021; Accessed 2021 June 4]. Available from: https://hospital. kau.edu.sa/Content-599-EN-38308

17. Wolters Kluwer. UpToDate. Evidence-based Clinical Decision Support. [Updated 2021; Accessed 2021 June 4]. Available from: https://www.wolterskluwer.com/en/solutions/uptodate

18. International Business Machines Corporation (IBM). Micromedex solutions. Watson Health. [Updated 2021; Accessed 2021 June 4]. Available from URL: https://www.ibm. com/watson-health/about/micromedex

19. National Institute for Health and Care Excellence. Guidelines on PPI. [Updated 2021; Accessed 2021 June 4]. Available from: https://www.evidence.nhs.uk/search?q=guidelines+on+ppi 
20. Mathew B, Mathew J, Kiran Y, Geethu C, Elsy Varghese S, Ivan T, et al. Study And Assessment Of Appropriateness In The Usage of Proton Pump Inhibitors In A Tertiary Care Teaching Hospital In South India. Indo Am J pharm 2015; 5: 2849-2856.

21. Thorsen K, Søreide JA, Kvaløy JT, Glomsaker T, Søreide K. Epidemiology of perforated peptic ulcer: age-and genderadjusted analysis of incidence and mortality. World $J$ Gastroenterol 2013; 19: 347-354.

22. Hoover JG, Schumaker AL, Franklin KJ. Use of intravenous proton-pump inhibitors in a teaching hospital practice. Dig Dis Sci 2009; 54: 1947-1952.

23. Barkun A, Bardou M, Marshall JK. Consensus recommendations for managing patients with nonvariceal upper gastrointestinal bleeding. Ann Intern Med 2003; 139: 843-857.

24. Daneshmend TK, Hawkey CJ, Langman MJS, Logan RF, Long RG, Walt RP. Omeprazole versus placebo for acute upper gastrointestinal bleeding: Randomized double blind controlled trial. BMJ 1992; 304: 143-147.

25. Lin HJ, Lo WC, Lee FY, Perng CL, Tseng GY. A prospective randomized comparative trial showing that omeprazole prevents rebleeding in patients with bleeding peptic ulcer after successful endoscopic therapy. Arch Intern Med 1998; 158: 54-58.
26. Akram F, Huang Y, Lim V, Huggan PJ, Merchant RA. Proton pump inhibitors: Are we still prescribing them without valid indications? Australas Med J 2014; 7: 465-470.

27. Abraham NS. Proton pump inhibitors: Potential adverse effects. Curr Opin Gastroenterol 2012; 28: 615-620.

28. Theurl I, Finkenstedt A, Schroll A, Nairz M, Sonnweber T, Bellmann-Weiler R, et al. Growth differentiation factor 15 in anaemia of chronic disease, iron deficiency anaemia and mixed type anaemia. Br J Haematol 2010; 148: 449-455.

29. McCarthy D. Iron deficiency anaemia due to proton pump inhibitors: clinical impact revealed. J Intern Med 2019; 285: 245-247.

30. Asdaq SMB, ALbasha M, Almutairi A, Alyabisi R, Almuhaisni A, Faqihi R, et al. Use of proton pump inhibitors: An exploration of awareness, attitude and behavior of health care professionals of Riyadh, Saudi Arabia. Saudi Pharm J 2021; 29: 713-718.

31. Mathioudakis A, Rousalova I, Gagnat AA, Saad N, Hardavella G. How to keep good clinical records. Breathe (Sheff) 2016; 12: 369-373. 